\title{
OBSERVATION ET EXPÉRIMENTATION SUR UN ESSAIM NIDIFIANT ARTIFICIELLEMENT A L'AIR LIBRE
}

\author{
PAR \\ Roger DARCHEN \\ (Station de Recherches Apicoles, Bures-sur-Yvette)
}

\section{Introduction.}

Ians une de ses publications, Bouviek (I906) signale les performances de deux essaims d'Apis mellifica qu'il a observés dans les jardins du Muséum d'Histoire Naturelle: les insectes sont capables d'établir des bâtisses de cire qui les protègent dans une certaine mesure contre les perturbations atmosphériques. Ils les orientent, les épaississent suivant les nécessités. Mais Bouvier note cependant que les Abeilles n'ont pas su s'abriter par un toit contre les pluies qui pénètrent assez aisément entre les rayons.

I,es faits curieux que signale Bouvier m'ont amené à rechercher quelles sont les possibilités psychiques des Abeilles constructrices au cours de la nidification à l'air libre ; mais le phénomène est assez rare clans nos régions. D'autre part, il fallait contrôler les facteurs agissant sur le comportenent constructeur des insectes : la température de l'air ambiant, l'anémométrie, l'éclairement, les supports... J'ai donc pris le parti d'expérimenter à l'intérieur d'une serre dont les dimensions sont les suivantes: $4,5 \mathrm{~m}$ de long sur $3 \mathrm{~m}$ de large et $2,5 \mathrm{~m}$ de haut. I,es résistances chauffantes de la serre ne sont utilisées que lorsque la température ambiante est trop basse (autour de Io ${ }^{\circ}$ ). On n’emploie pas d'éclairage spécial et il subsiste donc à l'intérieur une variation de la température et de l'éclairement a vec les nuits et les jours. Mais la serre est recouverte de lattes de bois afin d'éviter l'entrée d'une lumière solaire autre que diffuse (précaution essentielle).

Un essaim de I5 000 Abeilles environ est inséré dans une ruche sans cadre ; les Abeilles se fixent au couvre-cadres, qui mesure $50 \mathrm{~cm} \times 43 \mathrm{~cm}$, et construisent rapidement un rayon sur lequel elles s'attachent. A ce moment on soulève le support, la première bâtisse et 1'essaim. On les suspend au moyen d'un fil de fer à l'une des parties métalliques, à droite de l'axe longitudinal de la serre. 
J'ai utilisé deux essaims ; le premier m'a surtout permis de rechercher si les conditions de vie imposées n'étaient pas nuisibles à la bonne marche de la colonie, le deuxième m'a donné le moyen d'apprécier quelques affirmations de Bouvifr et d'effectuer des contre-expériences. Certes, je ne prétends pas que toutes les conclusions, établies seulement sur ces deux essaims, sont définitives mais elles pourront guider des travaux ultérieurs.

I,es Abeilles sont nourries dans la serre avec du sirop de sucre dans des boîtes de Petri garnies de billes de verre pour éviter la noyade et avec du pollen frais pulvérulent déposé dans des récipients voisins des premiers.

\section{La nidilication à l’air libre. Particularités.}

Au début de leur installation, les Abeilles forment une grappe imposante d'insectes enchevêtrés les uns dans les autres. MtYvir (I956) a remarqué que cette masse n'est pas inorganisée : une enveloppe dense, composée d'environ trois couches d'abeilles, entoure des chaînes cirières internes beaucoup plus lâches, sur lesquelles courent des ouvrières. Le centre de la grappe est formé d'insectes dont 1'âge est inférieur à I 9 jours, tandis que ceux qui entourent le trou de vol ont 2 I jours et ceux de l'enveloppe Ig à 25 jours. On distingue aussi un "trou de vol» deux à trois heures après l'installation de l'essaim en un point de l'enveloppe : c'est à cet endroit que les ouvrières entrent et sortent, comme l'a déjà vu l'auteur précédent.

Sur les premières bâtisses, l'essaim est assez populeux pour entourer constamment les cirières au travail à l'intérieur ; il ne se dissocie un peu qu'après des perturbations ou au moment des fortes chaleurs, lorsque la température dépasse $35^{\circ}$ à l'intérieur de la serre. Pendant les matinées les plus fraîches, on a tout le loisir d'observer à l'extérieur de la grappe ces couches d'Abeilles entremêlées qui semblent bien être aux Abeilles, ce qu'est l'involucre du nids aux Vespides. D'ailleurs, un certain nombre d'auteurs (Philips et DEmuTh, I9I4) (Wilson et Milum, I927), ont déjà signalé ce regroupement des insectes en amas serrés lorsque la température descend au-dessous de ${ }^{\circ} 8^{\circ} \mathrm{C}$ et surtout lorsqu'elle atteint $\mathrm{I}_{4}^{\circ} \mathrm{C}$. En regardant par dessous, on peut constater alors que l'enveloppe d'Abeilles est composée de zones circulaires concentriques (7 ou 8) ; entre elles, la densité des insectes reste beaucoup plus faible. Ainsi donc, même l'enveloppe extérieure de MEYFr pourrait se décomposer en plusieurs couches.

Au cours du temps, les bâtisses deviennent trop grandes pour que la population puisse les couvrir entièrement : en conséquence, les cirières se regroupent en différents secteurs suivant les besoins de l'évolution du nid. Dans la couche d'Abeilles externe on peut distinguer quelquefois des ouvrières dont l'abdomen est chargé de petites lamelles de cire. Des Abeilles se tiennent aussi en permanence fixées aux rayons ; d'autres se 
déplacent sans cesse sur les tranches des rayons en arrachant et en récollant des débris de cire, ou bien visitent les cellules les unes après les autres.

Dès le début, les débris de pollen, portés par les récolteuses s'accumulent sur un papier disposé au-dessous de l'essaim dans une zone bien déterminée et invariable, qui ne correspond pas obligatoirement aux lieux d'emmagasinage. D'ailleurs, les Abeilles, aux pattes chargées de pollen. rentrent toutes du même côté en perçant l'enveloppe formée par leurs congénères, attachées les unes aux autres. A cette occasion, quelques débris doivent s'échapper et tomber sur le plateau horizontal. Cette zone d'entrée du pollen correspond anx bords des rayons, mais ne se tronre pas obligatoirement du côté le plus rapproché de la source de pollen et de nectar: chez un essainn, je l'ai vu à l'opposé, chez 1'autre en face. Mais je ne puis dire si elle correspond au " trou de vol signalé par MEYER. Fst-elle reconnue grâce à une structure spéciale de la colonie et de son support ou bien à l'aide de repères pris dans l'environnement? Quelques expériences aisées permettent de répondre à cette question. Si l'on tourne horizontalenent l'essaim (fortement asymétrique) et son support de $\mathbf{I} 8 \mathbf{0}^{\circ}$, les Abeilles entrent néanmoins du même côté et les débris se trouvent dans la méme zone par rapport à l'environnement. Il semble donc que les repères extérieurs jouent un grand rôle dans le conditionnement des Abeilles récolteuses de pollen. Il faudrait cependant continuer encore ce genre d'expériences en tournant le support de $30^{\circ}, 45^{\circ}$ et $90^{\circ}$, en le transportant dans un autre endroit, ou bien en transformant l'aspect des lieux par des panneaux appropriés.

\section{A(-1.ivité ronstructrice}

Si l'on dispose alors un plateau horizontal recouvert d'une feuille de papier noir à $40 \mathrm{~cm}$ sous l'essaim au travail, des écailles et des débris de cire s'y accumulent en rangées parallèles, laissant entre eux des zones de papier presque vierges : ces bandes de débris correspondent aux rayons en construction. Le poids de ces déchets est certainement proportionnel à la quantité de cire construite. Les Abeilles ne viennent pas les chercher à nouveau pour développer leurs rayons et les laissent inutilisés. Pendant 7 à Io jours, la cire récoltée sur la feuille de papier noir est en quantité faible et mélangée à très peu de pollen. Puis, petit à petit, ce dernier se trouve en très grande quantité.

Chaque jour, les débris de cire accumulés ont été pesés jusqu'au moment où la quantité des débris de pollen devient si importante qu'elle perturbe trop notablement les mesures. En même temps, les températures étaient enregistrées par un thermomètre enregistreur. Pour le premier essaim (A) on n'a opéré des relevés qu'à 9 h et 20 h et pour le second (B). à $9 \mathrm{~h}, \mathrm{I}_{5} \mathrm{~h}$ et $2 \mathrm{I} \mathrm{h}$. I,es tableaux ci-dessous groupent les résultats qui, à 
première vue, semblent étranges puisque (A) a travaillé davantage le jour et (B) la nuit.

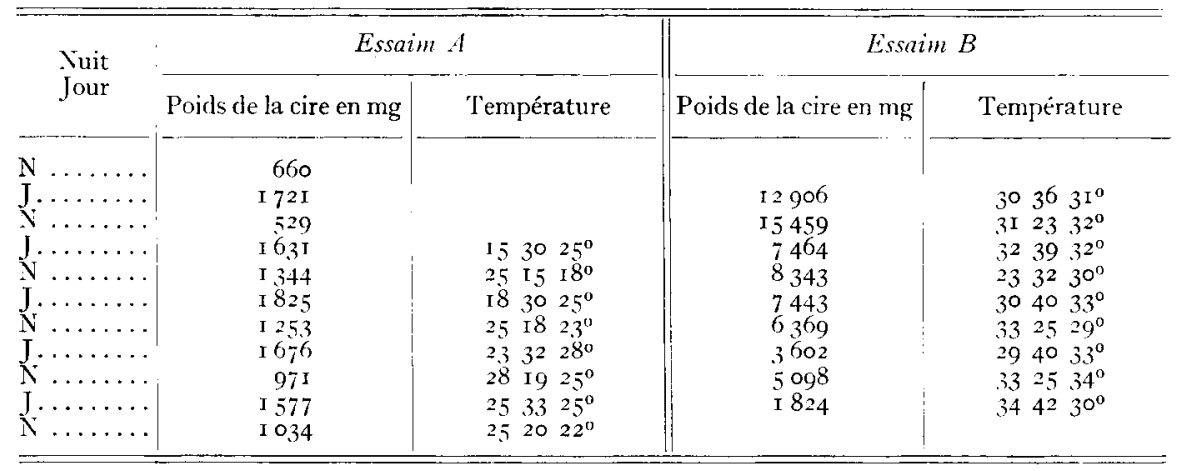

Io I'enregistrement des températures nous montre que le maximum de construction s'effectue lorsque la température externe oscille légèrement autour de $30^{\circ}$. Au-dessus de $30^{\circ}$, les nécessités de la régulation thermique dissocient les grappes plus ou moins proportionnellement à l'élévation de la température : les Abeilles quittent l'essaim, volent ici et là le long des vitres de la serre; les rayons de cire apparaissent démunis d'Abeilles sauf dans les zones occupées par le couvain.

$2^{\circ}$ I1 semble que pour les Abeilles, dont les bàtisses sont à 1'air libre, l'activité constructrice n'est pas soumise à un rythme nycthénéral.

\section{La résistance aux « intempéries ».}

I1 devenait possible de vérifier les assertions de Bouvier concernant les transformations effectuées par les Abeilles sur le plan général de leurs bâtisses afin d'affronter les intempéries de milieu ambiant.

Lorsqu'elles furent bien installées sur 9 rayons assez développés, j'ai dirigé, au moyen d'une soufflerie, un courant d'air d'une vitesse de 2 à $3 \mathrm{~m}$ par seconde dans une direction à peu près perpendiculaire au plan des orifices des cellules (fig. I). Immédiatement, après quelques heures d'adaptation, les grappes cirières se regroupèrent ; les rayons les plus éloignés de la soufflerie devinrent les plus peuplés. Dans le même temps, les derniers rayons croissaient plus rapidement que leurs devanciers plus proches de la soufflerie. I es faces des rayons exposés au courant d'air étaient privées d'ouvrières.

A la suite de Bouviêr, on aurait pu s'attendre à ce que les Abeilles fournissent des solutions spectaculaires aux problèmes proposés. Il semble, au contraire, que les insectes donnent une réponse purement mécanique et pas du tout "psychologique ": les grappes d'Abeilles se regroupent simplement et travaillent dans une zone abritée du vent.

Il suffit d'ailleurs d'arrêter la soufflerie pour que les grappes d'Abeilles 


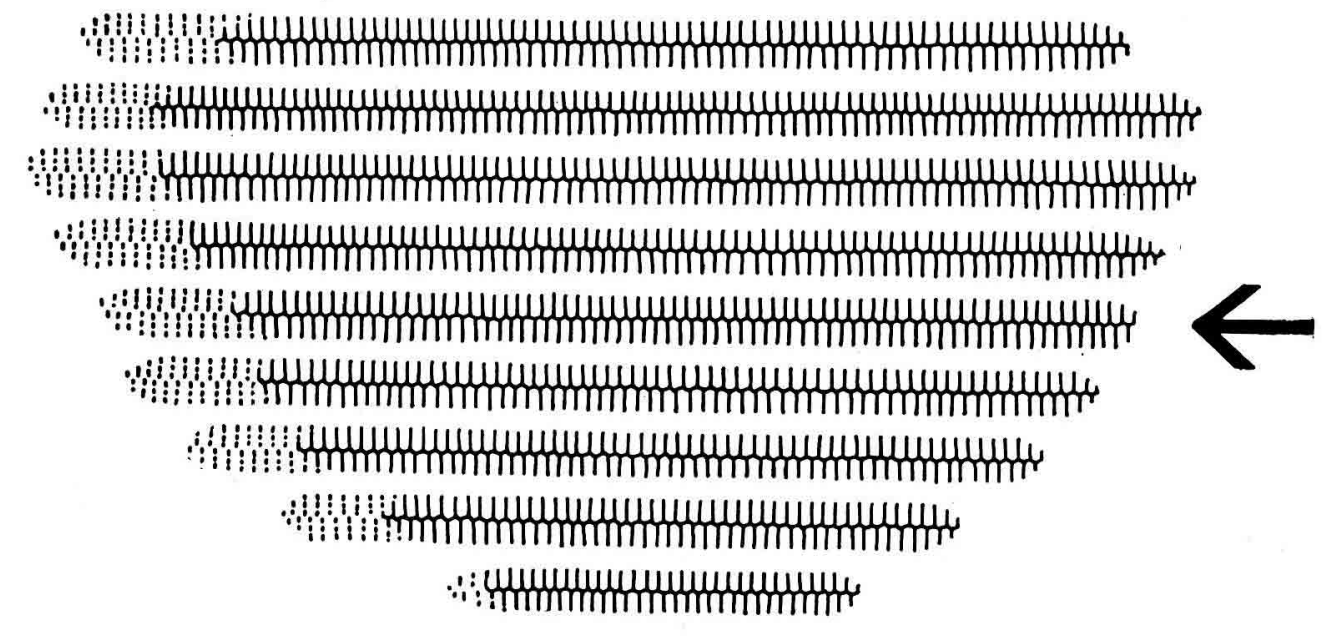

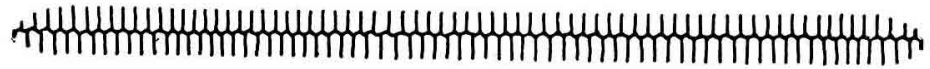

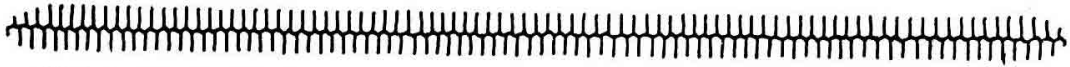

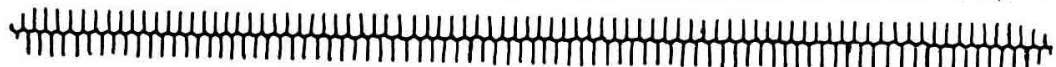

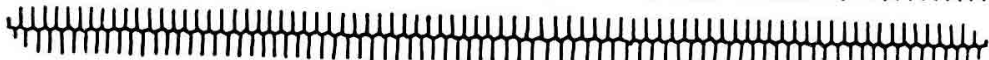

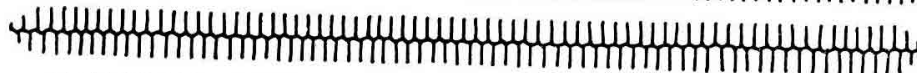

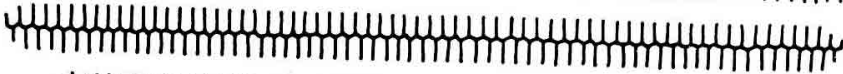

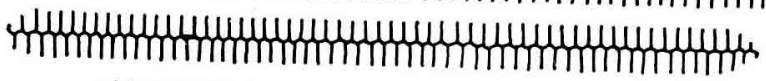

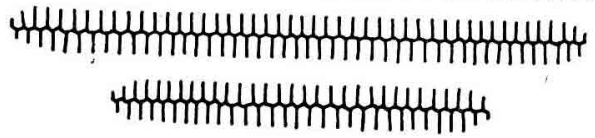

Firg. 1. 

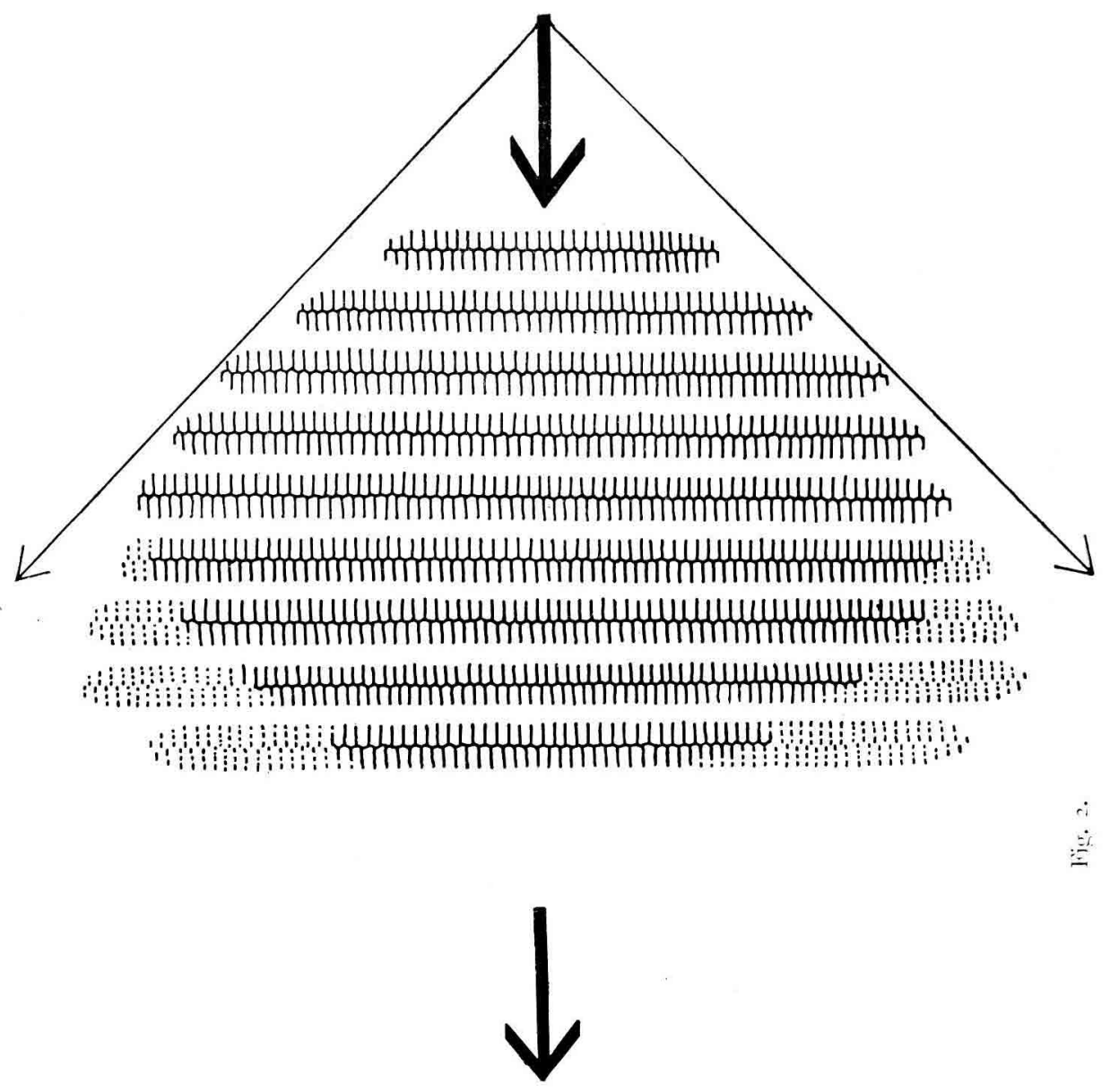

ת

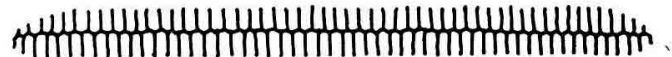

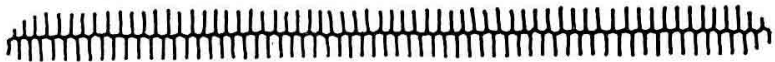

24

21

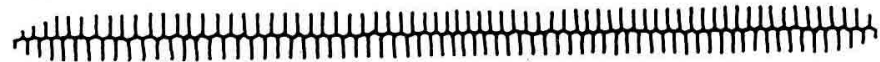

14

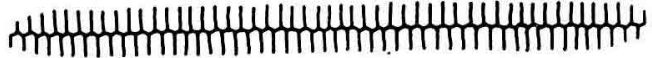

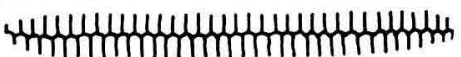


se répartissent unifornénent sur l'ensemble des ragons : les rayons abandonnés sont repenplés très rapidenuent.

On peut encore déplacer la soufflerie et envoyer son conrant d'air sur les borts des ravons construts. I ans ce cas, les cirières se replient à l'opposé et délaissent les portions des rayons les plus éventées : les bâtisses se développent donc anx endroits les moins perturbés (fig. 2).

Iinfun, signalons que l'agyessivité des Abeilles est presque nulle et qu'on peut à loisir les examiner de très près sans craincle nue piquire. I a woufferie ne les rend pas plus agressives, ce qui paraît étonnant.

\section{Comrlusion.}

Je n'ai fait que présenter nue technique de travail ct qu'aborder l'étude de quelques problèmes, mais on jeut constater déjà que bon nombre d'observations et expériences deviennent possibles : examens plus aisés des chaînes d'Abeilles et, pent-être des ouvrières au travail sur les opercules, less parois des cellules, etc., expérinentations sur la nidification dans divers biotopes artificiels ou dans des lieux et sur des supports les plus divers. Finfin, il semble que les possibilités d'adaptation des Abeilles à certaines conditions atmospliéricues soient très grandes, mais les insectes ne vont pas jusqu'à adopter une solution aussi complexe que celle qu'on observe parfois chez des mammifères supérieurs : en l'occurrence, elles ne construisent pas de " paravent ", elles ne ferment pas les anfractuosités et laissent passer les courants d'air. Il faudrait, $\vdots 1$ est vrai, diminuer la force du vent pour s'assurer si les réactions des insectes restent les mêmes ou s'adaptent alors. Cependant il semble bien que les essaims de Bourrik n'ont fait que se servir au mieux des irrégularités de leur support. Inutile de rechercher un "esprit de la ruche" (qui, dans nos expériences ne semble pas s'être démasqué.

\section{Réillmé.}

Après avoir indiqué une méthode d'observation d'essaims nidifiant en plein vent, l'auteur montre que, dans les conditions de ses expériences, les Abeilles produisent des bâtisses, suivant la température ambiante (dont 1'optimun oscille autour de $\left.30^{\circ}\right)$, mais non suivant le nycthémère. D'autre part, les insectes ne sont pas capables d'édifier des paravents de cire ou de boucher les anfractuosités qui laissent passer les courants d'air, créés artificiellement par une soufflerie : les solutions adoptées semblent purement mécaniques. On note encorc que les butineuses de pollen entrent dans les bâtisses par une zone privilégiée, reconnue au moyen de l'environnement, que 1'agressivité des insectes est presque nulle et que les débris de cire, recueillis sur un plateau horizontal au-dessous de l'essaim ne sont pas utilisés. 


\section{REFERENCES BIBLIOGRAPHIQUES}

Bouvier (E. L.). - Nouvelles observations sur la nidification des Abeilles à l'air libre. Ann. Soc. Ent. Fr., 75, 429-444, I9o6.

Lecomte (J.). - Les facteurs de l'agressivité chez l'Abeille. C. R. Acad. Sc., 232, $1376-8$, I951.

MEYER (W.). - Arbeitsteilung im Bienenschwarm. Insectes Sociaux, 3, 2, 303-324, I956.

PHI,IPS (E. F.), DEMuTh (G. S.). - The temperature of the Honeybee cluster in Winter. Bul. U. S. Dep. Agr., 98, I-I6, Igr4.

WrisoN (H. F.), Mru, UM (V. G.). - Winter protection for the honeybee colony. Res. Bul. Wis. Agr. Exp. Sta., 75, r-47, 1927. 